\title{
APPLICATION OF SIMULINK IN THE IMPLEMENTATION OF CALCULATION ALGORITHMS FOR CONTINUOUS WAVELET TRANSFORM ON A DIGITAL SIGNAL PROCESSOR
}

\author{
Dmitry V. Thuravov ${ }^{1}$, Andrey B. Stepanov ${ }^{2}$ \\ ${ }^{1}$ Synopsys SPb LLC, Prof. Popov Str., 23-D, Saint-Petersburg 193376, Russia \\ ${ }^{2}$ The Bonch-Bruevich Saint-Petersburg State University of Telecommunications \\ Bolshevikov prosp. 22-1, Saint-Petersburg 193232, Russia
}

The paper is received on May 7, 2019

\begin{abstract}
The paper describes the technology of implementing calculation algorithms for continuous wavelet transform on a digital signal processor using Simulink. The basic concepts of wavelets and continuous wavelet transform are given. Three calculation algorithms for continuous wavelet transform are considered: first, on the basis of a discrete wavelet transform, second, using a cross-correlation function and third, using a fast Fourier transform. The paper presents Simulink models that allow describing the calculation algorithms of continuous wavelet transform at the level of logical block diagrams, as well as the results of implementing these algorithms on Texas Instruments TMS320C5515 Evaluation Module (EVM). A comparative analysis of the wavelet spectrograms obtained in MATLAB and output to the LCD display of the debug board is performed.
\end{abstract}

Keywords: wavelet, continuous wavelet transform, digital signal processor, Simulink, MATLAB.

\section{Introduction}

Wavelets are a generalized name for families of basis functions with zero integral value. Such functions are widely used in the processing of signals of different nature due to the features of localization along the time axis and scaling in the frequency domain. These features allow the use of wavelets for time-frequency analysis of signals. Each family of basis functions contains a maternal wavelet, a shift along the time axis the scaling of which forms a set of basis functions. The maternal wavelet can be selected from a significant set of existing functions or, if necessary, can be synthesized to analyze a particular type of signal and to identify distinctive 
information features in it. Such wavelet features allow them to be used to accurately identify the time of appearance and disappearance of frequency components in the analyzed signal. Mathematically, this process is reduced to a continuous wavelet transform (CWT) over the signal.

The implementation of calculation algorithms for continuous wavelet transform on digital signal processors (DSPs) or programmable logic integrated circuits (FPGAs) is a complex task and requires significant time from the developer. Therefore, the technology of accelerated design of digital signal processing (DSP) systems with application of continuous wavelet transform can be of special interest.

The design of DSP systems generally consists of 3 stages:

1) Developing the mathematical apparatus of the designed system.

2) Modeling the system using specialized mathematical packages. MATLAB system can act as such a package.

3) Implementation of the system.

MATLAB contains tools that allow not only modeling DSP systems, but also a significant reduction of implementation time. Such tools are MATLAB Coder and Simulink Coder. MATLAB Coder allows us to obtain code for implementing DSP systems on FPGA or DSP based on user-written m-files. Simulink Coder generates code based on Simulink models. The processes of modeling and implementing in this case are combined.

Let us consider the possibility of using Simulink when implementing calculation algorithms for continuous wavelet transform on a digital signal processor.

The algorithms described below and Simulink models can also be used to generate HDL code for FPGAs.

\section{Continuous wavelet transform}

The formula for the continuous wavelet transform of the function $f(t)$ has the following form:

$$
W(a, b)=\frac{1}{\sqrt{a}} \int_{-\infty}^{\infty} f(t) \overline{\psi\left(\frac{t-b}{a}\right)} d t
$$


where $\psi(t)$ is wavelet; parameter $a$-determines the size of the wavelet; $b$-specifies a shift along the time axis, so that $a, b \in R, a \neq 0$; The horizontal bar indicates complex conjugation.

The results of continuous wavelet transform calculation can be visualized in the form of a two- or three-dimensional graph - a wavelet-spectrogram. The threedimensional wavelet spectrogram has the following axes: along the abscissa axis, the time shift is measured, along the ordinate axis the scale is measured, along the applicate axis the values of wavelet coefficients are measured. On a two-dimensional graph, the magnitude of the wavelet coefficients is determined by the color gradient.

The main advantage of continuous wavelet transform is the possibility to obtain information about the frequency of individual signal components and their localization in time. Therefore, the continuous wavelet transform has become widely used in the detection of information signs in biomedical (electroencephalogram, electrocardiogram, etc.), seismic and other signals.

As for disadvantages of the continuous wavelet transform, it is necessary to note its redundancy, which, as a rule, leads to an increase in the requirements for the element base on which calculation algorithms are implemented.

\section{Digital signal processor and debug board}

In this paper, calculation algorithms for continuous wavelet transform are implemented on a digital signal processor by Texas Instruments TMS320C5515. This device belongs to processors with a fixed point, has a classic architecture and combines high performance and low power consumption, which allows it to be used in the implementation of mobile digital signal processing systems. The processor speed is $120 \mathrm{MHz}$. The processor has two MAC blocks (multiply-accumulate).

The processor is installed on the TMS320C5515 Evaluation Module (EVM) debug board, which has the following features:

- 32-bit programmable low power stereo codec.

- Color LCD display.

- $\quad$ Line inputs and outputs.

- $\quad$ USB 2.0 port. 
- 10 user buttons.

- $\quad$ The ability to connect memory cards.

Mentioned features and pre-installed processor characteristics can be used to process audio, biomedical and seismic signals, so EVM is a good approach for CWT research.

\section{Technology of accelerated design of digital signal processing systems}

In order to increase the speed of designing DSPS with a CWP on a digital signal processor, it is proposed to use the C-code generation technology based on the Simulink model at the implementation stage. Let us consider the basic stages of design:

1) Stating the problem.

2) Developing the mathematical apparatus of the designed system, in which a continuous wavelet transform is applied. At this stage, the mother wavelet is also selected from a set of existing wavelets or a new one is synthesized and adapted to the analyzed signal.

3) Loading the signal and wavelet into the MATLAB system. The advantage of this package is a wide range of tools for modeling digital signal processing systems. In addition, all three of its main parts (the MATLAB core, the Simulink block modeling subsystem and the Toolbox collection) include elements for operating wavelets.

4) Creating Simulink-model system

5) Setting parameters of Simulink-model system

6) Analysis of modeling results

7) Setting parameters for generating C-code. At this stage, you can select the target device, as well as the optimization parameters.

8) Generatung C-code, getting the source and header files.

9) Creating a project in an integrated development environment.

10) Adding the generated files to the project.

11) Configuring the compilation and linking parameters.

12) Making the necessary corrections. The generated code is oriented to the processor. For the use of peripherals, additions are necessary. 
13) Building the model.

14) Functional test.

15) Loading the binary file into the processor memory.

\section{Calculation algorithms the continuous wavelet transform}

The paper proposes three calculation algorithms for continuous wavelet transform: on the basis of discrete wavelet transform (DWT); on the basis of cross correlation function (CCF) and using a fast Fourier transform (FFT). Let us consider each of these algorithms in more detail.

A. CWT calculation algorithms based on DWT

The discrete wavelet transform allows decomposition of the analyzed signal into approximating $A$ and $D$ specifying coefficients. The results of the calculation of CWTs are related to the values of the detailing coefficients obtained with DWT by the following formula [5]:

$$
W(a, b)=W\left(2^{j}, 2^{j} k\right)=D_{j, k},
$$

where $j$ - level of decomposition, $k$ - discrete normalized time.

In order to perform the CWT, it is proposed to use the Simulink model (Fig. 1) to calculate the decomposition coefficients of DWT with the settings for multilevel decomposition of the signal.

It includes Signal To Workspace blocks intended for exporting the received coefficients to the MATLAB workspace; Signal From Workspase1 block for loading a signal from the workspace; blocks Discrete FIR Filter - LPF and HPF decomposition; Downsample blocks - decimators.

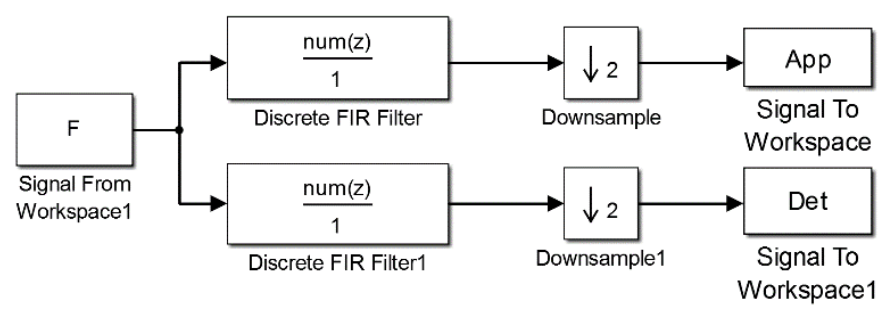

Fig. 1. Simulink-model of CWT calculation algorithm based on DWT 
Fig. 2 shows the results of CWT obtained at 5 levels of its decomposition. Investigating the obtained graphs, we can conclude that this algorithm allows us to calculate the coefficients of the CWT with the help of DWT, but the obtained wavelet spectrogram is of little use for further analysis. Therefore, this algorithm will not be implemented on the DSP.

a)

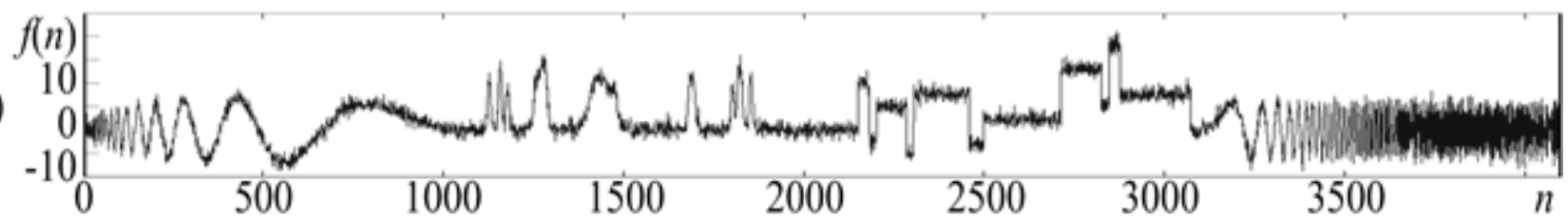

b)

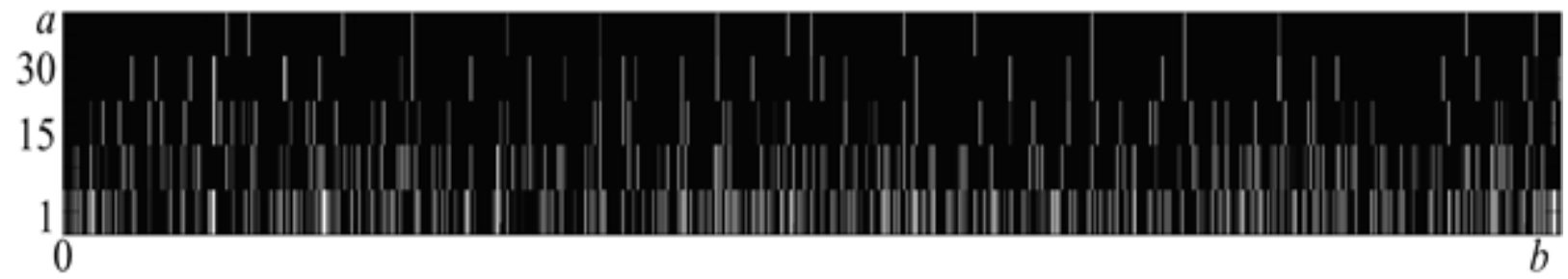

Fig. 2. Results of CWT: original signal $a$ ), wavelet-spectrogram $b$ )

B. Algorithm based on $C C F$

In this paper, CWT calculation algorithm based on $\mathrm{CCF}$ is proposed. In formula (1) continuous time $t$ is used. To implement the CWT on the DSP, this formula is to be presented in a discrete form. Let us perform the replacement:

$$
\psi_{a}(t)=\psi(-t / a) .
$$

Then formula (1) takes the form:

$$
W(a, b)=\frac{1}{\sqrt{a}} \int_{-\infty}^{\infty} f(t) \overline{\psi_{a}(b-t)} d t .
$$

We use the Morlet wavelet for modeling. The wavelet function is even and real, so expressions (2) and (3) can be rewritten in the following form [5]:

$$
\begin{gathered}
\psi_{a}(t)=\psi(t / a), \\
W(a, b)=\frac{1}{\sqrt{a}} \int_{-\infty}^{\infty} f(t) \psi_{a}(t-b) d t .
\end{gathered}
$$

Let us move from a continuous time to a discrete one, we get: 


$$
W(p T, m T)=\frac{1}{\sqrt{p T}} \sum_{n=-\infty}^{\infty} f(n T) \psi_{p T}(n T-m T),
$$

where $n T$ is discrete values of time instant; $f(n T)$ is a discrete signal to be analyzed; $\psi_{p T}(n T)$ is discrete wavelet function, depending on the scaling factor; $W(p T, m T)$ is discrete values of wavelet coefficients.

Passing from discrete to discrete normalized time for the signal, we get:

$$
W(p, m)=\frac{1}{\sqrt{p}} \sum_{n=0}^{N-1} f(n) \psi_{p}(n-m),
$$

where $N$ is the number of samples in the signal and the wavelet function.

Formula (4) is a cross-correlation function without taking into account the $1 / N$ factor and allows determining the mutual correlation of the signal $f(n)$ and the wavelet function $\psi_{p}(n)$.

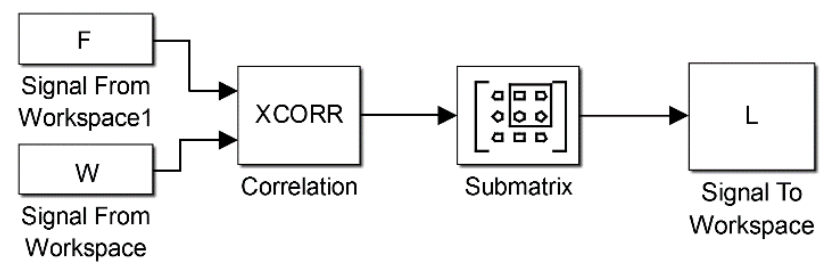

Fig. 3. Simulink-model of CWT calculation algorithm based on CCF

Fig. 3 shows the Simulink model, which allows calculating the CWT using formula (4). It contains Signal To Workspace blocks for inputting a signal and a wavelet from the working area; Correlation block which performs direct calculation by the CCF formula; a Submatrix block that allocates a submatrix from the output matrix of the Correlation block; Signal To Workspace block for output of the waveletspectrogram to the working area of MATLAB. Fig. 5, $b$ shows the waveletspectrogram obtained with CWT sinusoids (Fig. 5, $a$ ) on the basis of CCF algorithm. C. Algorithm based on FFT

In this paper, an algorithm for calculating CWT using FFT is proposed. Thus, the wavelet coefficients are calculated not in the time domain directly by formula (4), but in the frequency domain [8]. For this purpose, the Fast Correlation Theorem is 
used. Using the discrete Fourier transform (DFT) formula and formula (4), we represent the DWT of the wavelet coefficients in the following form:

$$
W_{F F T}(p, k)=\sum_{m=0}^{N-1} W(p, m) \mathrm{e}^{-i \frac{2 \pi}{N} m k}, W_{F F T}(p, k)=\sum_{m=0}^{N-1}\left[\frac{1}{\sqrt{p}} \sum_{n=0}^{N-1} f(n) \psi_{p}(n-m)\right] \mathrm{e}^{-i \frac{2 \pi}{N} m k},
$$

where $k$ is the discrete normalized frequency; $W_{F F T}(p, k)-N$-point DFT.

Let us multiply and divide the right-hand side by the twiddle factor. We put the term $1 / \sqrt{p}$ outside the brackets:

$$
\begin{gathered}
W_{F F T}(p, k)=\frac{1}{\sqrt{p}} \sum_{m=0}^{N-1} \sum_{n=0}^{N-1}\left[f(n) \psi_{p}(n-m)\right] \mathrm{e}^{-i \frac{2 \pi}{N} m k} \frac{\mathrm{e}^{-i \frac{2 \pi}{N} n k}}{-i \frac{2 \pi}{N} n k} . \\
W_{F F T}(p, k)=\frac{1}{\sqrt{p}} \sum_{n=0}^{N-1} f(n) \mathrm{e}^{-i \frac{2 \pi}{N} n k} \sum_{m=0}^{N-1} \psi_{p}(n-m) \mathrm{e}^{i \frac{2 \pi}{N} k(n-m)} .
\end{gathered}
$$

The DFT image of the sequence $\psi_{p}(n)$ can be determined using the expression:

$$
\Psi_{p}(k)=\sum_{n=0}^{N-1} \psi_{p}(n) \mathrm{e}^{-i \frac{2 \pi}{N} n k} .
$$

Taking into account the Delay Theorem:

$$
\Psi_{p}(k)=\sum_{n=0}^{N-1} \psi_{p}(n-m) \mathrm{e}^{-i \frac{2 \pi}{N} n k} \mathrm{e}^{i \frac{2 \pi}{N} m k} .
$$

If $l=n-m$ then:

$$
\Psi_{p}(k)=\sum_{(m+l)=0}^{N-1} \Psi_{p}(n-m) \mathrm{e}^{-i \frac{2 \pi}{N} k(n-m)}, \Psi_{p}(k)=\sum_{m=-l}^{N-1} \Psi_{p}(n-m) \mathrm{e}^{-i \frac{2 \pi}{N} k(n-m)}
$$

We divide the given sum into two:

$$
\Psi_{p}(k)=\sum_{m=-l}^{-1} \Psi_{p}(n-m) \mathrm{e}^{-i \frac{2 \pi}{N} k(n-m)}+\sum_{m=0}^{N-1} \psi_{p}(n-m) \mathrm{e}^{-i \frac{2 \pi}{N} k(n-m)} .
$$

Since $m$ is a nonnegative number, the left sum in the formula is 0 . Let us find the complex conjugate value of the right and left parts: 


$$
\overline{\Psi_{p}(k)}=\overline{\left[\sum_{m=0}^{N-1} \psi_{p}(n-m) \mathrm{e}^{-i \frac{2 \pi}{N} k(n-m)}\right]} .
$$

Since the Morlet wavelet is a real function, then:

$$
\overline{\Psi_{p}(k)}=\sum_{m=0}^{N-1} \Psi_{p}(n-m) \mathrm{e}^{i \frac{2 \pi}{N} k(n-m)} .
$$

By putting this expression into formula (5), we obtain:

$$
W_{F F T}(p, k)=\frac{1}{\sqrt{p}} \sum_{n=0}^{N-1} f(n) \mathrm{e}^{-i \frac{2 \pi}{N} n k} \overline{\Psi_{p}(k)} .
$$

Since $\overline{\Psi_{p}(k)}$ does not depend on the summation variable $n$, then:

$$
W_{F F T}(p, k)=\frac{1}{\sqrt{p}} \overline{\Psi_{p}(k)} \sum_{n=0}^{N-1} f(n) \mathrm{e}^{-i \frac{2 \pi}{N} n k}
$$

Under the summation sign we have the DFT image of the signal $f(n)$ under investigation. The final formula looks as follows:

$$
W_{F F T}(p, k)=\frac{1}{\sqrt{p}} F(k) \overline{\Psi_{p}(k)} .
$$

Thus, by performing the inverse discrete Fourier transform (IDFT) of the function $W_{F F T}(p, k)$, we obtain the desired value of the wavelet coefficients. In the calculation of DFT images, the FFT algorithm with time decimation is applied.

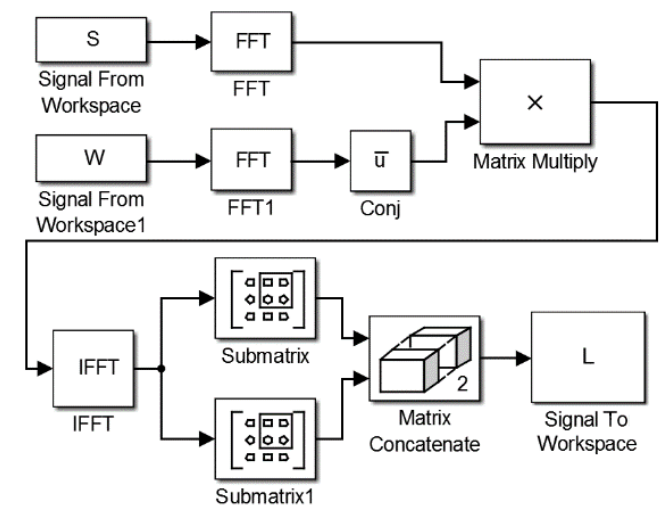

Fig. 4. Simulink-model of the algorithm for CWT calculation based on FFT

The Simulink algorithm model (Fig. 4) includes: Signal To Workspace blocks for signal input and wavelet; FFT blocks for calculating the DFT images of the signal 
and the wavelet; Conj block carrying out complex conjugation of the input signal; Matrix Multiply block, allowing to calculate the element-by-element product of two input matrices; the IFFT block calculating the IDFT; Submatrix, Matrix Concatenate blocks for correct display of wavelet spectrogram and time alignment; Signal To Workspace block for output of the wavelet-spectrogram to the working area of MATLAB. Fig. 5, c) shows the wavelet-spectrogram obtained with CWT sinusoids (Fig. 5, a) based on the algorithm with FFT.

\section{Implementation of CWT calculation algorithms}

Based on the Simulink models, C-code generation was performed. First, the Simulink model was modified to work with a fixed point, which is necessary for the selected DSP. Further, in Simulink Coder the target processor was chosen, the settings for generation and optimization of $\mathrm{C}$-code were set. The files received during the generation were imported into the project created in the integrated development environment of Code Composer Studio. After making additions to the code for working with peripherals, an output file was received. Fig. 5 shows photographs of the display of the debug board, obtained during CWT calculation using the algorithm with CCF (Fig. 5, $d$ ) and FFT (Fig. 5, $d$ ). As follows from the figures, the best result is achieved with the use of the CCF-based algorithm: the wavelet spectrogram on the display is analogous to the wavelet spectrogram obtained in Simulink modeling.

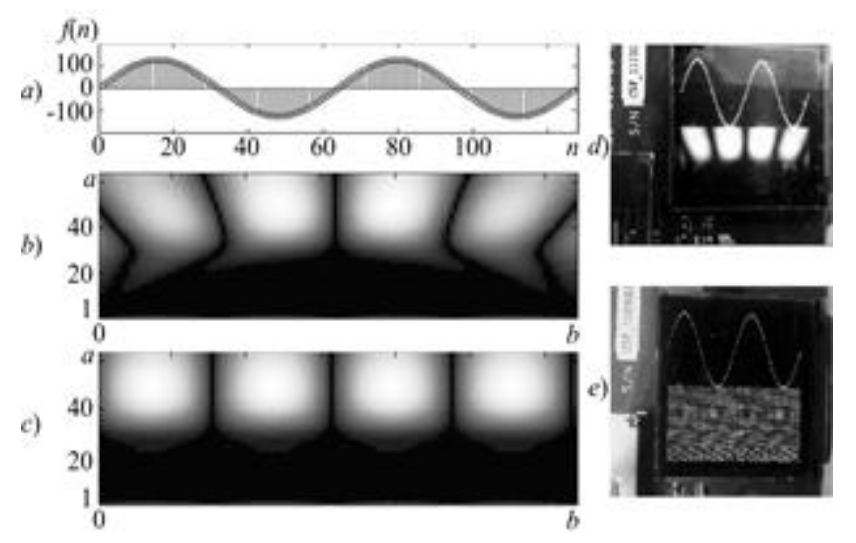

Fig. 5. Results of modeling and implementation of CWT calculation algorithms

\section{Conclusion}

Analyzing the results obtained during the implementation of CWT calculation algorithms on DSP, we can make the following conclusions: 
1) Continuous wavelet transform can be calculated using algorithms based on DWT, CCF and FFT.

2) Algorithm based on DWT does not allow obtaining a wavelet-spectrogram suitable for signal analysis.

3) Algorithm with $\mathrm{CCF}$, allows obtaining a wavelet-spectrogram, approximate to the results of modeling.

4) The technology of accelerated design on the basis of Simulink-model allows accelerating the process of implementation of DSP system with CWT.

5) The disadvantages of the technology of accelerated design are the need to supplement $\mathrm{C}$-code for working with the periphery and the difficulty of reading the generated C-code.

\section{References}

1. A.I. Solonina, S.M. Arbuzov. Tsifrovaya obrabotks signalov. Modelirovanie v MATLAB [Digital Signal Processing. Modeling in MATLAB]. St. Petersburg BHV-Petersburg Publ., 2008. (In Russian)

2. I. Daubechies. Ten Lectures on Wavelets. SIAM, 1992.

3. C. Blatter. Wavelets - Eine Einfuhrung. A.K. Peters Ltd., 1998.

4. N.K. Smolentsev. Osnovy teorii veyvletov. Veivlety v MATLAB. [Fundamentals of the Theory of Wavelets. Wavelets in MATLAB]. Moscow, DMK-Press Publ., 2014.

5. G.G. Stark. Ispolzovanie veivletov dlya tsifrovoy obrabotki signalov [The Use of Wavelets for DSP]. Moscow, Technosphera Publ., 2007. (In Russian)

6. A.B. Stepanov. The Application of Neural Network and Spline Wavelet Models in the Electroencephalogram Analysis Automation Process, Proceedings of FRUCT Conf., Apr. 2016, pp. 321-327.

7. A.B. Stepanov. Neural network model of wavelets for the continuous wavelet transform. Proc. of ICCTPEA Conf., Jun.-Jul. 2014, pp. 177-178.

8. E. Ifeachor, B. Jervis. Digital Signal Processing: a practical approach. Pearson Education Limited, 2002.

\section{For citation:}

Dmitry V. Zhuravov, Andrey B. Stepanov. Application of Simulink in the Implementation of Calculation Algorithms for Continuous Wavelet Transform on a Digital Signal Processor. Zhurnal Radioelektroniki - Journal of Radio Electronics. 2019. No.5. Available at http $/ /$ jre.cplire.ru/jre/may19/10/text.pdf 